\title{
ON UNIVALENT MAPPINGS BY SOLUTIONS OF LINEAR ELLIPTIC PARTIAL DIFFERENTIAL EQUATIONS $\left({ }^{1}\right)$
}

\author{
BY \\ PAUL W. BERG
}

1. In general, a differentiable homeomorphism need not have a nonvanishing Jacobian. Indeed all of the first partial derivatives of such a mapping may vanish at a point. In the case of plane conformal mappings this is not so, as is well-known. One can even assert for the Jacobian of a univalent conformal mapping at a point a positive lower bound which depends only on the distance of the point and its image from the boundaries of the original and image domains (Koebe's Theorem). Similar statements hold for univalent mappings by solutions of general first order linear elliptic systems of partial differential equations.

In $1936 \mathrm{H}$. Lewy [5] showed that even for plane harmonic mappings (mappings by pairs of not necessarily conjugate harmonic functions) univalence implies the nonvanishing of the Jacobian. This statement, however, cannot be made quantitative. It is easy to construct univalent harmonic mappings of the unit disc onto itself which leave the origin fixed but have arbitrarily small Jacobian at the origin. Nevertheless, the weaker statement that the first partial derivatives of such a mapping do not all vanish at a point can be made quantitative. This was proved by E. Heinz in a recent paper [3]. The following theorem extends this result to mappings by solutions of general second order linear elliptic systems of partial differential equations with a common principal part.

THEOREM. Let the linear system

$$
\left\{\begin{array}{l}
A u_{x x}+2 B u_{x y}+C u_{y y}+a_{1} u_{x}+b_{1} u_{y}+c_{1} v_{x}+d_{1} v_{y}=0 \\
A v_{x x}+2 B v_{x y}+C v_{y y}+a_{2} u_{x}+b_{2} u_{y}+c_{2} v_{x}+d_{2} v_{y}=0
\end{array}\right.
$$

be elliptic $\left(A C-B^{2}>0\right)$ for $x^{2}+y^{2} \leqq 1$.

There exists a constant $\mu>0$, depending only on the coefficients, such that, if $u(x, y), v(x, y)$ is a solution of (1) for $x^{2}+y^{2}<1$, and $u=u(x, y), v=v(x, y)$ maps the disc $x^{2}+y^{2} \leqq 1$ homeomorphically onto $u^{2}+v^{2} \leqq 1$, leaving the origin fixed, then

$$
u_{x}^{2}+u_{y}^{2}+v_{x}^{2}+\left.v_{y}^{2}\right|_{(0,0)} \geqq \mu
$$

Received by the editors April 20, 1956.

(1) The research reported in this paper was sponsored by the Office of Naval Research. 
The coefficients of the principal part of (1) are assumed to have Höldercontinuous second partial derivatives, the remaining coefficients Hölder-continuous first partial derivatives for $x^{2}+y^{2} \leqq 1$.

For the special case

$$
\left\{\begin{array}{l}
u_{x x}+u_{y y}=0 \\
v_{x x}+v_{y y}=0
\end{array}\right.
$$

that is, for harmonic mappings, this statement reduces to Heinz' theorem. The proof, however, even in the harmonic case, is quite different.

The proof is direct. We wish to show that for the class of mappings considered the modulus of the gradient at the origin has a positive minimum. We prove first that for univalent mappings by solutions of (1) the gradient is nowhere zero, and the same is true of the limit of a sequence of such mappings, unless the limit is a constant. We then select a convergent minimizing sequence and show that the limit is not a constant.

It is worth pointing out that the theorem includes the case in which the components $u(x, y), v(x, y)$ of the mappings each satisfy the same single second order elliptic equation, or even different equations with a common principal part. The only other conditions imposed are topological. In particular, the mappings need not be quasi-conformal. If, however, quasi-conformality can be established as an additional property, the inequality of the theorem yields a lower bound for the Jacobian.

Some possible extensions of the theorem will be evident from the proof. Thus, for example, the conclusion of the theorem can be stated in the slightly stronger form: There exists for every $r<1$ a constant $\mu(r)>0$, depending only on $r$ and the coefficients, such that $u_{x}^{2}+u_{y}^{2}+v_{x}^{2}+v_{y}^{2} \geqq \mu(r)$ for $x^{2}+y^{2} \leqq r^{2}$. Also, mappings of more general domains, in particular multiply connected domains, can be discussed.

2. We observe first that it is sufficient to consider the special system

$$
\left\{\begin{array}{l}
\Delta u+a_{1} u_{x}+b_{1} u_{y}+c_{1} v_{x}+d_{1} v_{y}=0, \\
\Delta v+a_{2} u_{x}+b_{2} u_{y}+c_{2} v_{x}+d_{2} v_{y}=0,
\end{array}\right.
$$

since the general system (1) can be transformed into (3) by a smooth homeomorphism of the unit disc with itself which leaves the origin fixed (cf. [1, p. 79]).

It is convenient to introduce complex notation. Set $z=x+i y, w=u+i v$, $w(z)=u(x, y)+i v(x, y)$. Employing the formal differential operators

$$
\frac{\partial}{\partial z}=\frac{1}{2}\left(\frac{\partial}{\partial x}-i \frac{\partial}{\partial y}\right), \quad \frac{\partial}{\partial \bar{z}}=\frac{1}{2}\left(\frac{\partial}{\partial x}+i \frac{\partial}{\partial y}\right),
$$

we may write the system (3) in the form 


$$
\frac{\partial^{2} w}{\partial z \partial \bar{z}}+a \frac{\partial w}{\partial z}+b \frac{\partial w}{\partial \bar{z}}+c \frac{\overline{\partial w}}{\partial z}+d \frac{\overline{\partial w}}{\partial \bar{z}}=0,
$$

with complex-valued coefficients which are linear combinations of the coefficients of (3). We have also

$$
\begin{aligned}
u_{x}^{2}+u_{y}^{2}+v_{x}^{2}+v_{y}^{2} & =2\left(\left|\frac{\partial w}{\partial z}\right|^{2}+\left|\frac{\partial w}{\partial \bar{z}}\right|^{2}\right), \\
u_{x} v_{y}-u_{y} v_{x} & =\left|\frac{\partial w}{\partial z}\right|^{2}-\left|\frac{\partial w}{\partial \bar{z}}\right|^{2} .
\end{aligned}
$$

For univalent mappings the Jacobian (6) is either everywhere non-negative or everywhere nonpositive. We may assume that it is non-negative, so that

$$
\left|\frac{\partial w}{\partial z}\right| \geqq\left|\frac{\partial w}{\partial \bar{z}}\right|
$$

everywhere.

A function $\phi(z)$ which satisfies the inequality

$$
\left|\frac{\partial \phi}{\partial \bar{z}}\right| \leqq K|\phi|
$$

in a domain $D$ is said to be approximately analytic in $D$ with constant $K$. We shall use as a lemma a theorem of Bers, the "similarity principle," which states then

LemMA A. If $\phi(z)$ is approximately analytic with constant $K$ in a domain $D$,

$$
\phi(z)=e^{s(z)} f(z),
$$

where $f(z)$ is analytic in $D$, and $s(z)$ has a bound and Hölder-modulus of continuity which depend only on $K$.

The proof is given in $[1$, p. 171 (see also pp. 18, 25)].

We shall employ another theorem of Bers, namely

Lemma B. If $w(z)$ is a twice Hölder continuously differentiable solution of (4) in a neighborhood of $z_{0}$ and

$$
w(z)=O\left(\left|z-z_{0}\right|^{n}\right), \quad \frac{\partial^{2} w(z)}{\partial z \partial \bar{z}}=O\left(\left|z-z_{0}\right|^{n-1}\right)
$$

for some integer $n \geqq 1$, then there exist constants $\alpha, \beta$ such that

$$
w(z)=\alpha\left(z-z_{0}\right)^{n}+\beta\left(\overline{z-z_{0}}\right)^{n}+o\left(\left|z-z_{0}\right|^{n}\right),
$$




$$
\begin{aligned}
& \frac{\partial w(z)}{\partial z}=n \alpha\left(z-z_{0}\right)^{n-1}+o\left(\left|z-z_{0}\right|^{n-1}\right), \\
& \frac{\partial w(z)}{\partial \bar{z}}=n \beta\left(\overline{z-z_{0}}\right)^{n-1}+o\left(\left|z-z_{0}\right|^{n-1}\right) .
\end{aligned}
$$

This is a specialization of Lemma 4.1 of [2 (also see the remark on p. 487)].

Finally, we need a well-known statement based on a priori estimates due to Schauder.

LEMMA C. Every uniformly bounded sequence $\left\{w_{n}(z)\right\}$ of solutions of (4) in a domain $D$ contains a subsequence which converges normally $\left.{ }^{2}\right)$ in $D$ to a solution w(z). The first and second partial derivatives of the functions of the subsequence also converge normally to the corresponding derivatives of the limit.

The statement follows, by a standard argument, from Theorem 1 of [6, p. 265 (see also pp. 258, 269)].

It is necessary to remark that by a solution of (4) we mean a function with continuous second derivatives which satisfies the equation. It can be shown, however, that such a function possesses Hölder-continuous second derivatives (cf. [4]).

3. The proof of our theorem rests on four lemmas, the first two of which are essentially local in character.

LEMMA 1. Let $w(z)$ be a solution of (4) which is univalent in a neighborhood of a point $z_{0}$. Then $\left|(\partial w / \partial z)\left(z_{0}\right)\right|>0$.

Proof. If $w(z)$ is univalent and satisfies (4) in a domain $D$ containing $z_{0}$, then from (4) and (7)

$$
\left|\frac{\partial}{\partial \bar{z}} \frac{\partial w}{\partial z}\right| \leqq K\left|\frac{\partial w}{\partial z}\right|
$$

where

$$
K=\sup _{z \in D}(|a|+|b|+|c|+|d|) .
$$

Thus $\partial w / \partial z$ is approximately analytic in $D$. By Lemma A therefore

$$
\frac{\partial w(z)}{\partial z}=e^{s(z)} f(z)=\gamma\left(z-z_{0}\right)^{m}+o\left(\left|z-z_{0}\right|^{m}\right),
$$

where $m$ is a non-negative integer and $\gamma$ is a constant different from zero. From (7), (8) and (10) we have

(2) We say that a sequence of functions converges normally in a domain $D$ if the sequence converges uniformly in every subdomain whose closure is contained in $D$. 


$$
\frac{\partial w(z)}{\partial z}=O\left(\left|z-z_{0}\right|^{m}\right), \quad \frac{\partial w(z)}{\partial \bar{z}}=O\left(\left|z-z_{0}\right|^{m}\right), \quad \frac{\partial^{2} w(z)}{\partial z \partial \bar{z}}=O\left(\left|z-z_{0}\right|^{m}\right),
$$

and by integration we obtain

$$
w(z)-w\left(z_{0}\right)=O\left(\left|z-z_{0}\right|^{m+1}\right) .
$$

Set $n=m+1$. Then $w(z)-w\left(z_{0}\right)$ satisfies the hypothesis of Lemma $\mathrm{B}$, and we conclude

$$
\begin{aligned}
w(z) & =w\left(z_{0}\right)+\alpha\left(z-z_{0}\right)^{n}+\beta\left(\overline{z-z_{0}}\right)^{n}+o\left(\left|z-z_{0}\right|^{n}\right), \\
\frac{\partial w(z)}{\partial z} & =n \alpha\left(z-z_{0}\right)^{n-1}+o\left(\left|z-z_{0}\right|^{n-1}\right) .
\end{aligned}
$$

Comparing (10) and (11) we see that $n \alpha=\gamma \neq 0$, so that $\alpha \neq 0$.

If $n=1$ in equations (11) the statement of the lemma is established. We show that $n>1$ contradicts the hypothesis of univalence of $w(z)$.

We may assume that

$$
w(z)=z^{n}+\kappa \bar{z}^{n}+o\left(|z|^{n}\right),
$$

where $\kappa$ is a non-negative real number, since this form can be achieved by affine transformations of the $z$ - and $w$-planes, and these do not alter the univalence of the mapping. Set $z=r e^{i \theta}$. We have

$$
\operatorname{Re}\left\{w\left(r e^{i \theta}\right)\right\}=(1+\kappa) r^{n} \cos n \theta+o\left(r^{n}\right),
$$

and, in particular,

$$
\operatorname{Re}\left\{w\left(r e^{i(k / n) \pi}\right)\right\}=(1+\kappa) r^{n}(-1)^{k}+o\left(r^{n}\right),
$$

$k=0,1, \cdots, 2 n$. It follows that, for $\epsilon>0$ sufficiently small, and $r \leqq \epsilon$, the left side of (12) has the sign of $(-1)^{k}$. Hence, if $z$ traverses a Jordan curve $C$ which lies entirely within the disc $|z| \leqq \epsilon$ and has nonzero index with respect to the origin, $\operatorname{Re}\{w(z)\}$ undergoes at least $2 n$ alternations of sign.

Now, since $w(z)$ is a local homeomorphism we may choose $\epsilon$ and $\delta$ sufficiently small so that $w(z)$ has an inverse which maps $|w| \leqq \delta$ into $|z| \leqq \epsilon$. Let $C$ be the inverse image of the circumference $|w|=\delta / 2$. $C$ is a Jordan curve, of index one with respect to the origin, which lies entirely within the $\operatorname{disc}|z| \leqq \epsilon$. As $z$ traverses $C, \operatorname{Re}\{w(z)\}$ changes sign exactly twice. Thus we arrive at a contradiction unless $n=1$.

LeMma 2. Let $w_{n}(z) n=1,2, \cdots$ be univalent mappings satisfying (4) in a domain $D$. If the sequence $\left\{w_{n}(z)\right\}$ converges normally in $D$ to a solution $w(z)$ of (4), then either $w(z)$ is a constant or $|\partial w / \partial z|>0$ everywhere in $D$.

Proof. We observe first that, in consequence of Lemma $\mathrm{C}$, the normal convergence of $\left\{w_{n}(z)\right\}$ to $w(z)$ implies the normal convergence of $\left\{\partial w_{n}(z) / \partial z\right\}$, $\left\{\partial w_{n}(z) / \partial \bar{z}\right\}$ to $\partial w(z) / \partial z, \partial w(z) / \partial \bar{z}$, respectively. 
From (8) and (9) we see that

$$
\left|\frac{\partial}{\partial \bar{z}} \frac{\partial w_{n}}{\partial z}\right| \leqq K\left|\frac{\partial w_{n}}{\partial z}\right|
$$

where $K$ is independent of $n$. Thus the functions $\partial w_{n} / \partial z$ are uniformly approximately analytic in $D$. According to Lemma A therefore, there exist analytic functions $f_{n}(z)$ such that

$$
\frac{\partial w_{n}(z)}{\partial z}=e^{s_{n}(z)} f_{n}(z)
$$

where the functions $s_{n}(z)$ are uniformly bounded and equicontinuous in $D$. If the sequence $\left\{w_{n}(z)\right\}$ is replaced by a suitably chosen subsequence, the corresponding sequence $\left\{s_{n}(z)\right\}$ and hence also $\left\{f_{n}(z)\right\}$ will converge normally in $D$. Writing

$$
s(z)=\lim _{n \rightarrow \infty} s_{n}(z), \quad f(z)=\lim _{n \rightarrow \infty} f_{n}(z)
$$

we have

$$
\partial w(z) / \partial z=e^{s(z)} f(z) .
$$

According to Lemma 1 and equation (13), $\left|f_{n}(z)\right|>0$ everywhere in $D$, for all $n$. The limit of a sequence of analytic functions which have no zeros in a domain either has no zeros or vanishes identically (Hurwitz' Theorem). Thus, either $|f(z)|>0$ everywhere in $D$, or $f(z) \equiv 0$. If $|f(z)|>0$ everywhere, then, by (14), $|\partial w(z) / \partial z|>0$ everywhere. If $f(z) \equiv 0$, then $\partial w(z) / \partial z \equiv 0$, and, by (7), $\partial w(z) / \partial \bar{z} \equiv 0$, so that $w(z)$ is constant.

We remark that for harmonic mappings the proofs of Lemmas 1-2 are considerably simpler. If $w(z)$ is harmonic, then

$$
\partial w(z) / \partial z \text { and } \overline{\partial w(z) / \partial \bar{z}}
$$

are analy tic functions of $z$, so that Lemmas $A$ and $B$ are not needed. Lemma C follows immediately from the mean value property.

4. The following two lemmas refer to the global behavior of the mappings we consider. The first establishes a uniform connection between solutions of (4) and their boundary values; the second states an inequality for the boundary values of homeomorphisms of the unit disc onto itself.

Lemma 3. Let $w_{n}(z) n=1,2, \cdots$ be solutions of (4) for $|z|<1$, continuous and uniformly bounded for $|z| \leqq 1$. If the sequence $\left\{w_{n}(z)\right\}$ converges normally to zero in $|z|<1$, then the boundary values $\left\{w_{n}\left(e^{i \theta}\right)\right\}$ converge weakly (in $\left.L^{2}[0,2 \pi]\right)$ to zero.

Proof. We have to show that 


$$
\lim _{n \rightarrow \infty} \int_{0}^{2 \pi} w_{n}\left(e^{i \theta}\right) \sigma(\theta) d \theta=0
$$

for all $\sigma(\theta)$ in $L^{2}[0,2 \pi]$. Because of the uniform boundedness of $w_{n}\left(e^{i \theta}\right)$, it suffices to consider only functions $\sigma(\theta)$ which can be continued as twice continuously differentiable periodic functions with period $2 \pi$.

Define, for a fixed $\sigma(\theta)$,

$$
\phi_{R}(z)=\phi_{R}\left(r e^{i \theta}\right)=\frac{r^{3}}{R^{3}}(r-R) \sigma(\theta)
$$

for $|z| \leqq 1,1 / 2 \leqq R<1$. We have

$$
\begin{gathered}
\phi_{R}\left(R e^{i \theta}\right)=0, \quad \frac{\partial \phi_{R}}{\partial r}\left(R e^{i \theta}\right)=\sigma(\theta), \\
\left|\phi_{R}(z)\right|+\left|\frac{\partial \phi_{R}(z)}{\partial z}\right|+\left|\frac{\partial \phi_{R}(z)}{\partial \bar{z}}\right|+\left|\frac{\partial^{2} \phi_{R}(z)}{\partial z \partial \bar{z}}\right| \leqq M,
\end{gathered}
$$

where $M$ is independent of $z$ and $R$.

Applying Green's theorem to the disc $|z| \leqq R$, referring to (16), we obtain,

$$
\int_{0}^{2 \pi} w_{n}\left(R e^{i \theta}\right) \sigma(\theta) R d \theta=4 \iint_{|z| \leq R}\left(w_{n} \frac{\partial^{2} \phi_{R}}{\partial z \partial \bar{z}}-\phi_{R} \frac{\partial^{2} w_{n}}{\partial z \partial \bar{z}}\right) d x d y .
$$

From (4),

$$
\begin{aligned}
\iint_{|z| \leqq R} \phi_{R} \frac{\partial^{2} w_{n}}{\partial z \partial \bar{z}} d x d y & \\
= & -\iint_{|z| \leqq R} \phi_{R}\left(a \frac{\partial w_{n}}{\partial z}+b \frac{\partial w_{n}}{\partial \bar{z}}+c \frac{\overline{\partial w_{n}}}{\partial z}+d \frac{\overline{\partial w_{n}}}{\partial \bar{z}}\right) d x d y .
\end{aligned}
$$

Integration by parts yields, referring again to (16),

$$
\begin{aligned}
\iint_{|z| \leqq R} \phi_{R} \frac{\partial^{2} w_{n}}{\partial z \partial \bar{z}} d x d y= & \iint_{|z| \leqq R}\left[w_{n} \frac{\partial}{\partial z}\left(\phi_{R} a\right)+w_{n} \frac{\partial}{\partial \bar{z}}\left(\phi_{R} b\right)\right. \\
& \left.+\bar{w}_{n} \frac{\partial}{\partial \bar{z}}\left(\phi_{R} c\right)+\bar{w}_{n} \frac{\partial}{\partial z}\left(\phi_{R} d\right)\right] d x d y .
\end{aligned}
$$

Set

$$
M^{\prime}=\sup _{|z| \leqq 1}\left(|a|+|b|+|c|+|d|+\left|\frac{\partial a}{\partial z}\right|+\left|\frac{\partial b}{\partial \bar{z}}\right|+\left|\frac{\partial c}{\partial \bar{z}}\right|+\left|\frac{\partial d}{\partial z}\right|\right) .
$$

Then from (19) we get

$$
\left|\iint_{|z| \leqq R} \phi_{R} \frac{\partial^{2} w_{n}}{\partial z \partial \bar{z}} d x d y\right| \leqq M M^{\prime} \iint_{|z| \leqq R}\left|w_{n}\right| d x d y .
$$


We also have

$$
\left|\iint_{|z| \leqq R} w_{n} \frac{\partial^{2} \phi_{R}}{\partial z \partial \bar{z}} d x d y\right| \leqq M \iint_{|z| \leqq R}\left|w_{n}\right| d x d y .
$$

Combining (18), (20) and (21), and letting $R \rightarrow 1$, we obtain finally

$$
\left|\int_{0}^{2 \pi} w_{n}\left(e^{i \theta}\right) \sigma(\theta) d \theta\right| \leqq 4 M\left(1+M^{\prime}\right) \iint_{|z| \leqq 1}\left|w_{n}\right| d x d y .
$$

The hypothesis of the lemma implies that the right side of (22) converges to zero as $n \rightarrow \infty$, from which (15) follows.

LemMa 4. Let $w(z)$ be a homeomorphism of the disc $|z| \leqq 1$ with $|w| \leqq 1$. Then

$$
\left|\int_{0}^{2 \pi / 3} w\left(e^{i \theta}\right) d \theta\right|+\left|\int_{2 \pi / 3}^{4 \pi / 3} w\left(e^{i \theta}\right) d \theta\right|+\left|\int_{4 \pi / 3}^{2 \pi} w\left(e^{i \theta}\right) d \theta\right| \geqq \frac{\pi}{3} .
$$

Proof. The points $1, e^{2 \pi i / 3}, e^{4 \pi i / 3}$ divide the circumference $|z|=1$ into three equal arcs each of which is mapped biuniquely by $w(z)$ onto an arc of $|w|=1$. One at least of the image arcs must have length not exceeding $2 \pi / 3$. Call this $\operatorname{arc} A$, and suppose that it is the image of $z=e^{i \theta}, 0 \leqq \theta \leqq 2 \pi / 3$. $B y$ the mean value theorem

$$
\int_{0}^{2 \pi / 3} w\left(e^{i \theta}\right) d \theta=\frac{2 \pi}{3} w_{0}
$$

where $w_{0}$ is a point in the convex hull of $A$. Hence

$$
\left|\int_{0}^{2 \pi / 3} w\left(e^{i \theta}\right) d \theta\right|=\frac{2 \pi}{3}\left|w_{0}\right| \geqq \frac{2 \pi}{3} \cdot \frac{1}{2}=\frac{\pi}{3},
$$

and, a fortiori, (23) holds.

5. The proof of our theorem is now immediate. Let $H$ denote the class of mappings satisfying the hypothesis of the theorem, and define

$$
\mu=\inf _{w(z) \in H} 2\left(\left|\frac{\partial w}{\partial z}(0)\right|^{2}+\left|\frac{\partial w}{\partial \bar{z}}(0)\right|^{2}\right) .
$$

Let $\left\{w_{n}(z)\right\}$ be a sequence of mappings in $H$ such that

$$
\lim _{n \rightarrow \infty} 2\left(\left|\frac{\partial w_{n}}{\partial z}(0)\right|^{2}+\left|\frac{\partial w_{n}}{\partial \bar{z}}(0)\right|^{2}\right)=\mu .
$$

According to Lemma $C$ the sequence may be so chosen that it converges normally to a solution $w(z)$, and we have 


$$
\mu=2\left(\left|\frac{\partial w}{\partial z}(0)\right|^{2}+\left|\frac{\partial w}{\partial \bar{z}}(0)\right|^{2}\right) .
$$

By Lemma 2 either $\mu>0$ or $w(z)$ is a constant. If $\mu>0$ the theorem is proved. If $w(z)$ is a constant, then, since $w_{n}(0)=0$ for all $n, w(z) \equiv 0$. Hence, by Lemma 3 , the boundary values $w_{n}\left(e^{i \theta}\right)$ converge weakly to zero in $L^{2}[0,2 \pi]$. But, from Lemma 4,

$$
\lim _{n \rightarrow \infty}\left(\left|\int_{0}^{2 \pi / 3} w_{n}\left(e^{i \theta}\right) d \theta\right|+\left|\int_{2 \pi / 3}^{4 \pi / 3} w_{n}\left(e^{i \theta}\right) d \theta\right|+\left|\int_{4 \pi / 3}^{2 \pi} w_{n}\left(e^{i \theta}\right) d \theta\right|\right) \geqq \frac{\pi}{3} .
$$

Thus if $w(z)$ is a constant we arrive at a contradiction.

\section{BIBLIOGRAPHY}

1. L. Bers, Theory of pseudo-analytic functions, Lecture notes (mimeographed), New York University, 1953.

2. L. Bers, Local behaviour of solutions of general linear elliptic equations, Comm. Pure Appl. Math. vol. 8 (1955) pp. 473-496.

3. E. Heinz, Über die Lösungen der Minimalflächengleichung, Nachrichten der Akademie der Wissenschaften in Göttingen. Mathematisch-Physikalische Klasse (1952) pp. 51-56.

4. E. Hopf, Über den funktionalen, insbesondere den analytischer Character der Lösungen elliptischer Differential-gleichungen zweiter Ordnung, Math. Zeit. vol. 34 (1931) pp. 191-233.

5. H. Lewy, On the non-vanishing of the Jacobian in certain one-to-one mappings, Bull. Amer. Math. Soc. vol. 42 (1936) pp. 689-692.

6. J. Schauder, Über lineare elliptische Differentialgleichungen zweiter Ordnung, Math. Zeit. vol. 38 (1934) pp. 257-282.

STANFORD UNIVERSITY, Stanford, Calif. 